\title{
MEASURED RADIO WAVE BEHAVIOR OF A PARTIALLY OPEN DRAIN IN URBAN ENVIRONMENT
}

\author{
S.Y. Lim*, C.C. Pu, and Y. H. Liew ${ }^{\dagger}$ \\ * Sunway University, Malaysia \\ gracel@sunway.edu.my
}

Keywords: Propagation measurement, drain, radio wave, electromagnetic, wireless communications.

\begin{abstract}
This paper attempts to analyze the propagation characteristics of radio signal inside a partially open drain environment using an empirical approach. The proposed research topic concerns a unique situation in several Asian countries because the drainage systems in these countries differ notably from those in the US and Europe in that the former are partially open on the top while the latter are primarily below ground and covered. Measurement results are reported for one drain located on Sunway University campus at 2.4 and $5.8 \mathrm{GHz}$. The differences between the measurement results at the aforementioned two frequencies are discussed. These measurement results serve as a useful indicator as to how signal might behave differently in partially enclosed spaces, such as those where partially open drains exist, especially in an urban environment.
\end{abstract}

\section{Introduction}

The successful worldwide implementation of cellular mobile radio systems has resulted in an exponential growth in demand for wireless communications in the past decades. The general trend in the development of modern wireless communication systems includes the use of higher data rates (broader frequency band), as well as propagation in more complex environments [1]. Since wireless communications rely on electromagnetic (EM) waves to carry messages, a detailed and thorough comprehension of the pattern of radio waves propagation in complex environment contributes significantly to the effectual design and implementation of reliable wireless communication systems.

A review of the cellular communication systems shows that radio cell sizes have been shrinking - from large $(\sim$ hundreds of kilometres) to small ( $\sim$ tens of meters or less) [2]. There is a continuing growing need to accumulate the propagation knowledge in these small cells such as pico- and femto- cells because they have been increasingly used in cellular telephony systems. Picocells, for instance, are used in cellular telephony for high-traffic areas such as railway stations, office buildings and airports [5].
In a well-developed urban environment, various objects (be it moving or static) such as buildings, trees, vehicles, or partially open drains cause scattering that interferes with the transmission and reception of messages, which in turn could create cold spots - areas that lack connection. As a matter of fact, the various objects that form part of urban propagation environments can affect the propagation characteristics significantly in the frequency range above $300 \mathrm{MHz}$ [4]. In order to better understand the subject of urban propagation, partially open drain environment has been singled out as the object of studies in this paper using an empirical approach. The payoff of this research can help to establish dependable wireless communication networks in urban as well as other complex propagation environments.

\section{Measurement system setup}

A similar concept as that of the Data Acquisition Unit (DAU) reported in [3] has been assembled for field measurement campaigns. In this work, the assembled DAU is being utilized to collect a series of measurement data at 2.4 and $5.8 \mathrm{GHz}$. Figure 1 shows the block diagram of the measurement system.

Tx

$\mathrm{Rx}$

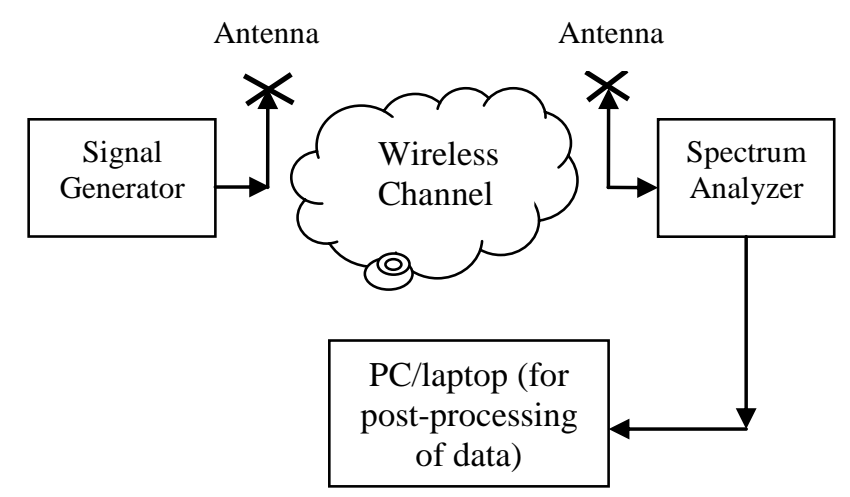

Figure 1: The setup of the measurement system.

On the transmitting end, one signal generator is employed while on the receiving end, a spectrum analyzer is utilized. These two pieces of equipments are the Rohde \& Schwarz SMB100A RF and Microwave Signal Generator and Rohde \& Schwarz FSH Handheld Spectrum Analyzer respectively. The 
signal generator can transmit RF signal in the range of $9 \mathrm{kHz}$ to $6 \mathrm{GHz}$ while the spectrum analyzer can pick up signal that lies between $100 \mathrm{kHz}$ to $6 \mathrm{GHz}$. The frequency bands chosen for this research are $2.4 \mathrm{GHz}$ and $5.8 \mathrm{GHz}$ because these two frequency bands have wide applications predominantly among the deployment of wireless Local Area Network (WLAN).

As for the antennas, two sets of dipole antennas at 2.4 and 5.8 $\mathrm{GHz}$ have been stationed at the transmitter (Tx) and receiver (Rx) locations. They are the $8 \mathrm{dBi}$ high performance omnidirectional wireless LAN antennas (HyperLink Technologies HGV-2409U) at $2.4 \mathrm{GHz}$; and the $8 \mathrm{dBi}$ ISM/UNII band omnidirectional wireless LAN antenna (HyperLink Technologies HG5808U) at $5.8 \mathrm{GHz}$. At 2.4 $\mathrm{GHz}$, the vertical beamwidth is $15^{\circ}$ and the horizontal beamwidth is $360^{\circ}$; while at $5.8 \mathrm{GHz}$, the vertical beam width is $16^{\circ}$ and the horizontal beam width is $360^{\circ}$. The Tx and $\mathrm{Rx}$ antennas are oriented for vertical-vertical (VV) polarization.

\section{The drain structure}

In this work, the focus is given to drains in urban environment. The drainage systems in the US and Europe differ notably from those in the South East Asian countries like Malaysia in that the former are primarily below ground and covered while the latter are partially open on the top. A survey of the drain structure worldwide shows that these two types of drain make up the majority of drains in the world. In particular, partially open drain is especially common in Malaysia and other South East Asian countries like Singapore, Indonesia, and Thailand. Besides, they can also be found in other Asian cities such as Taipei, Hong Kong, and India. The propagation characteristics in this type of partially open drain environments have not been studied in the past and will be the focus of this work. Figure 2 illustrates an example of the common U-shaped drain in Malaysia [6].

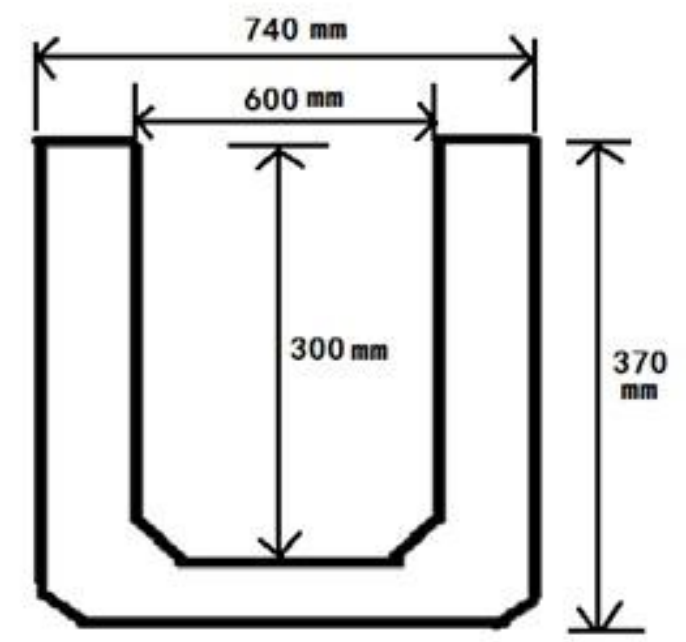

Figure 2: Standard size U-shaped drain in Malaysia.
In addition to Figure 2, Figure 3 presents the actual drain where the measurement campaign was conducted during sunny day. This drain is located on the campus of Sunway University, nearby its Gate 2.

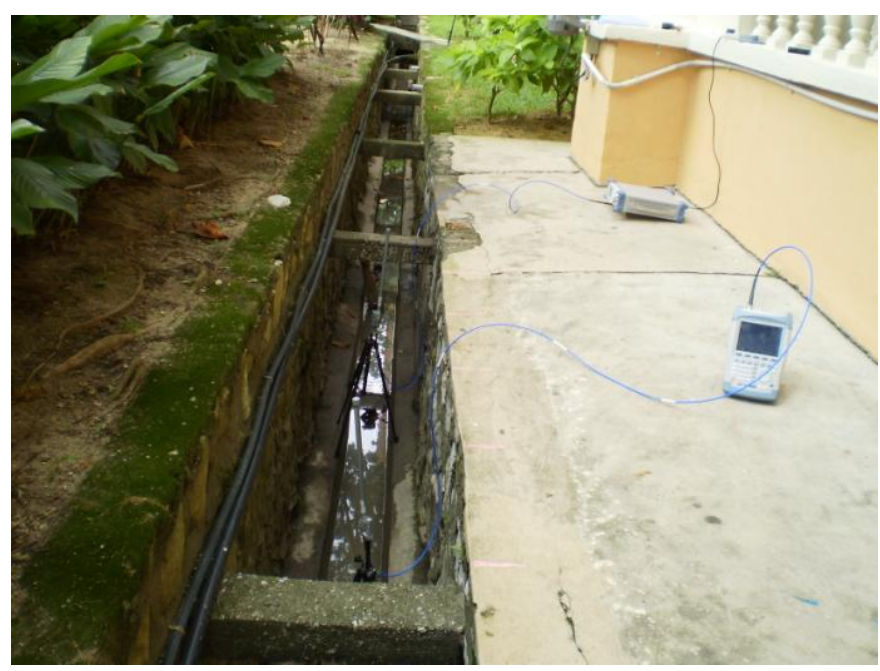

Figure 3: The actual measurement site.

\section{Measurement results and conclusion}

A series of measurements have been conducted at the measurement site as shown in Figure 3 using the Data Acquisition Unit assembled and described in Section 2. The received power due to a transmitter (Tx) along a route inside the drain is measured at $50 \mathrm{~cm}$ intervals. Both the transmitting and receiving antennas are dipoles and vertical polarization is considered.

The Tx and Rx antennas are mounted on a tripod and their heights are set to be equal, $123 \mathrm{~cm}$. These two antennas are placed inside the drain, as shown in Figure 3, with the first Rx location being placed $200 \mathrm{~cm}$ away from the Tx. The subsequent Rx locations are $50 \mathrm{~cm}$ away from each other, and at every $\mathrm{Rx}$ location, the receiver power is sampled multiple times in a 5-second period. These sampled values are being averaged and displayed on the Spectrum Analyzer. They are then recorded and designated as the received power at each specific Rx location.

Measurement results are validated by repeated measurements. Specifically, one week after the first measurement was conducted, the second measurement was carried out again. Figure 4 shows the first and second measurement results inside the drain for $2.4 \mathrm{GHz}$ while Figure 5 displays the first and second measurement results inside the same drain for 5.8 $\mathrm{GHz}$. We can observe from these two figures that the two repeated measurement results at both 2.4 and $5.8 \mathrm{GHz}$ exhibit the same trend. The difference between these two measurements is small with a mean (average) error of $2.57 \mathrm{~dB}$ and a standard deviation of $4.46 \mathrm{~dB}$ at $2.4 \mathrm{GHz}$; and a mean (average) error of $-0.40 \mathrm{~dB}$ and a standard deviation of 1.84 $\mathrm{dB}$ at $5.8 \mathrm{GHz}$ 


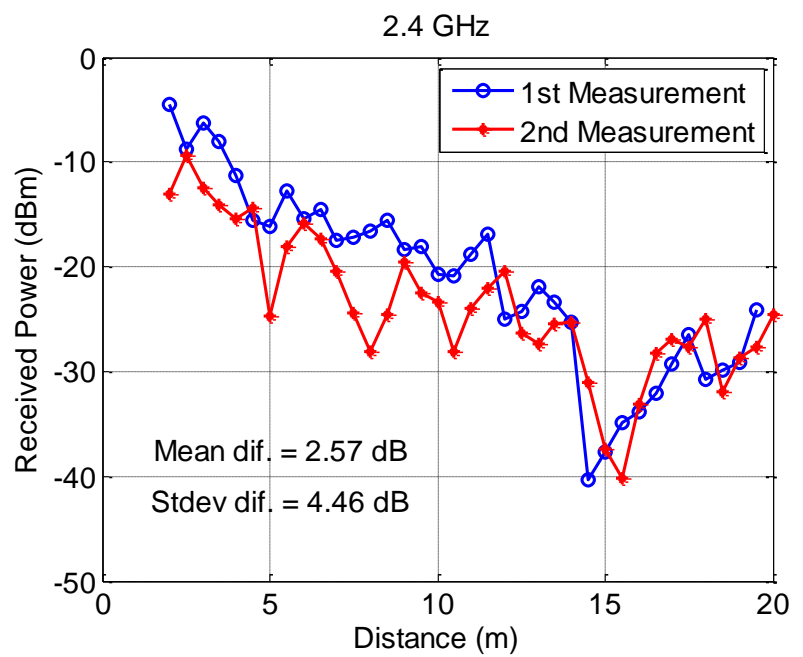

Figure 4: The measured received power for VV-polarization at $2.4 \mathrm{GHz}$.

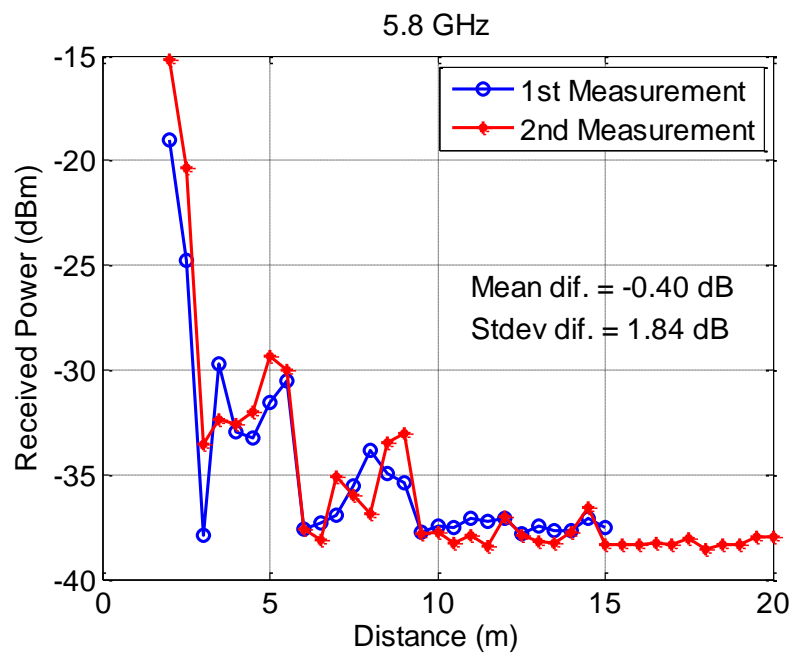

Figure 5: The measured received power for $\mathrm{VV}$-polarization at $5.8 \mathrm{GHz}$.

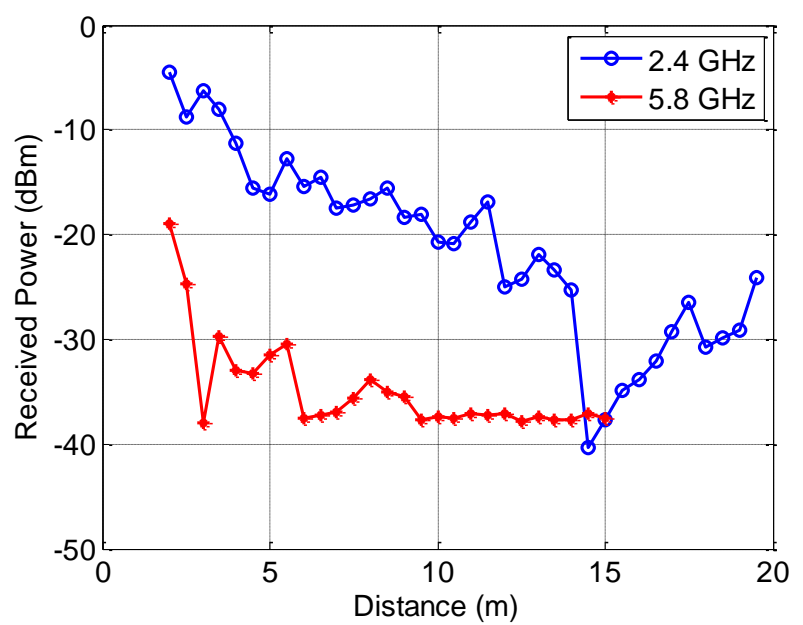

Figure 6: The comparison of measurement results between the two frequencies.
In addition to Figures 4 and 5, Figure 6 is included whereby the first measurement result of 2.4 and $5.8 \mathrm{GHz}$ are plotted together. There is an average difference of approximately 13 $\mathrm{dB}$ between the two frequencies in this figure, although the Tx power from the signal generator was set to the maximum in both cases. We can conclude that the $13 \mathrm{~dB}$ path loss added to the $5.8 \mathrm{GHz}$ is due to higher frequency losses and other reasons like the environment effects. Also, we can conclude that at lower frequency, which is $2.4 \mathrm{GHz}$ in this case, signal can propagate stronger and travel farther. The obtained path loss characteristics are useful for understanding radio propagation in pico- or femto-cells that have great potential in increasing the capacity and reducing the operational cost for wireless communication systems.

\section{Acknowledgements}

This work was supported by the Sunway University Internal Research Grant, INT-SCT-0111-04.

\section{References}

[1] M. F. Iskander, and Z. Yun, "Propagation prediction models for wireless communication systems," IEEE Trans. Microwave Theory and Tech., vol. 50, no. 3, pp. 662-673, March 2002.

[2] S. Y. Lim, Radio wave propagation measurement and modeling in wireless communication environments, $\mathrm{PhD}$ dissertation, the University of Hawaii at Manoa, United States, December 2010.

[3] S. Y. Lim, Z. Yun, J. M. Baker, N. Celik, H. Youn, and M. F. Iskander, "Propagation modeling and measurement for a multi-floor stairwell," IEEE Antennas and Wireless Propagation Letters, vol. 8, pp. 583-586, 2009.

[4] W. M. Smith, Urban propagation modeling for wireless systems, PhD dissertation, Stanford University, United States, February 2004.

[5] S. R. Saunders, and A. Aragon-Zavala, Antennas and propagation for wireless communication systems, $2^{\text {nd }}$ Edition, John Wiley \& Sons, Ltd, pp. 283-329, 2007.

[6] http://www.api.com.my/product_u-shape.asp 